\title{
Genomic Alterations Are Enhanced in Placentas from Pregnancies with Fetal Growth Restriction and Preeclampsia: Preliminary Results
}

\author{
Tal Biron-Shental ${ }^{a, c}$ Reuven Sharony ${ }^{a-c}$ Atalia Shtorch-Asor ${ }^{b}$ Meirav Keiser ${ }^{b}$ \\ Dana Sadeh-Mestechkina, ${ }^{a}$ Ido Laish ${ }^{b, c}$ Aliza Amiel ${ }^{b, d}$ \\ ${ }^{a}$ Department of Obstetrics and Gynecology, and ${ }^{b}$ Genetics Institute, Meir Medical Center, Kfar Saba, ${ }^{c}$ Sackler School \\ of Medicine, Tel Aviv University, Tel Aviv, and d Faculty of Life Science, Bar Ilan University, Ramat Gan, Israel
}

\section{Key Words}

Copy number variations · Fetal growth restriction · Placenta · Preeclampsia

\begin{abstract}
Fetal growth restriction (FGR) secondary to placental insufficiency and preeclampsia (PE) are associated with substantially increased childhood and adult morbidity and mortality. The long-term outcomes are related to placental aberrations and intrauterine programming. Advances in microarray technology allow high-resolution, genome-wide evaluation for DNA copy number variations - deletions and duplications. The aim of our study was to demonstrate the usefulness of microarray testing in FGR placentas. Using Affymetrix GeneChip for chromosomal microarray (CMA), we analyzed 10 placentas from pregnancies with FGR attributed to placental insufficiency; 5 with FGR below the 5 th percentile and 5 from the 5 th to $<10$ th percentiles. All fetuses had normal anomaly scans and karyotypes. We also analyzed 5 third-trimester placentas from pregnancies complicated by PE with severe features and 5 from PE without severe features, all with appropriately grown fetuses. The results were compared to 10 placentas from uncomplicated pregnancies with healthy neonates. CMA analysis identified more genomic al-
\end{abstract}

(C) 2016 S. Karger AG, Basel

$1661-8769 / 16 / 0066-0276 \$ 39.50 / 0$ terations in FGR $(p<0.05)$ and in PE $(p<0.05)$ placentas than in healthy controls. There was a correlation to the severity of FGR and PE. The genomic alterations were below the resolution of normal karyotyping. The altered genes are related to adult human height, stress reactions and to cellular migration, differentiation and adhesion. Though very preliminary, our data support evaluating FGR and PE placentas using CMA. Larger data sets are needed for further evaluation of the findings and their clinical implications.

(c) 2016 S. Karger AG, Basel

The intrauterine environment has a tremendous influence on fetal well-being as well as on life-long health [Barker, 2006; Palloto and Kilbride, 2006]. The placenta has an important role in the pathophysiology of pregnancy and its outcomes. The long-term effects are related to placental aberrations and intrauterine programming [Barker, 2006; Durnwald and Landon, 2013; Patti, 2013; Ross and Desai, 2013]. Fetal growth restriction (FGR) secondary to placental insufficiency and preeclampsia (PE), which is characterized by new onset hypertension and proteinuria related to abnormal placentation, are associated with substantially increased childhood and adult morbidity and mortality [Sibai, 2005; Sibai and Dekker, 2005].

\section{KARGER 125}

E-Mail karger@karger.com

www.karger.com/msy
Tal Biron-Shental, MD

Department of Obstetrics and Gynecology, Meir Medical Center

Tschernihovsky Street 59

Kfar Saba 44282 (Israel)

E-Mail shentalt@inter.net.il 
Table 1. Clinical characteristics

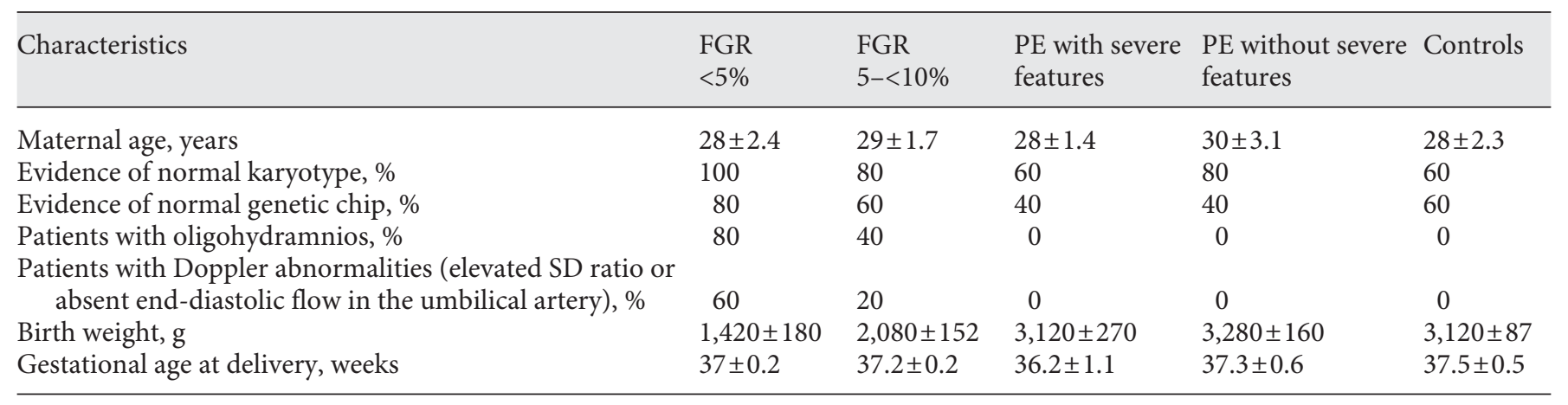

Values are means $\pm \mathrm{SD}$, unless indicated otherwise.

Interestingly, these 2 complications are attributed to abnormal placentation and share similar features, but do not always occur together. Although it is well established that the placenta contributes to both FGR and PE, the pathogenesis leading to these conditions is not fully understood.

Advances in microarray technology allow high-resolution, genome-wide evaluation of DNA copy number variations (CNVs) - both deletions and duplications and opens new directions for research connecting genetics to abnormal placentation [Founds et al., 2009; Ganesamoorthy et al., 2013; Madeleneau et al., 2015; Rabaglino et al., 2015]. It is a significant tool for identifying genomic alterations leading to diseases. Moreover, it allows disease pathway evaluation. CNVs are a result of genomic abnormalities, aging process or cancer progression, but importantly, they are also a normal part of cell biology and normal placentation. Therefore, even in normal placentas there are CNVs. Their prevalence and the involved genes may reflect specific placental insults reflected by different clinical characteristics [Lupski, 2013].

Previous studies have shown that alterations in genes may be related to placental invasion, immune response, inflammatory reactions, etc. Some changes occur as early as the first trimester of pregnancy and may be causal, while others may develop later and are a secondary response to stress-related conditions during pregnancy [Founds et al., 2009; Louwen et al., 2012].

CNVs are probably a normal aspect of the trophoblastic biology of the placenta. Specific changes occur during pregnancy to allow placental invasion and to systematically regulate genome changes during pregnancy [Hannibal et al., 2014]. Therefore, placenta-based investigations of CNVs are important for understanding FGR and PE and the genes and pathways related to these pregnancy-related complications. Even in cases of normal karyotypes and normal genetic chip findings, there are microarray data that are considered as normal variants and therefore not reported. However, they may contain important information regarding placental evaluation of pregnancy-related complications such as FGR and PE, attributed to abnormal placentation. The goal of this study was to demonstrate the usefulness of microarray testing in these placentas.

\section{Materials and Methods}

\section{Study Groups and Sample Collection}

Placental biopsies were obtained from 30 patients with welldated pregnancies who delivered during the third trimester. All fetuses had normal anomaly scans and good prenatal care with a low probability of a known phenotype or genetic syndrome. Some had normal karyotype and normal genetic chip results after amniocentesis performed during pregnancy. All participants had normal results for cytomegalovirus, toxoplasmosis, rubella, and herpes. Upon delivery and based on early neonatal evaluation, no genetic abnormalities were suspected. Placentas from fetuses with known abnormal karyotypes or abnormal genetic chips were not included in the study. Only placentas that had a normal genetic chip were included, to ensure looking at $\mathrm{CNVs}$ which were not typical of any known genetic abnormality.

Placental biopsies were collected from 5 patients who had fetuses with FGR $<5$ th percentile for the gestational age, based on locally accepted charts [Dollberg et al., 2005], and 5 placentas with FGR from the 5 th to $<10$ th percentiles. Based on clinical characteristics, FGR was attributed to placental insufficiency (table 1). These were compared to 5 placentas from uncomplicated pregnancies with healthy neonates. Another set of placentas was from pregnancies complicated by $\mathrm{PE}$, defined as blood pressure above 140/90 and 24-hour proteinuria above $300 \mathrm{mg}$. Five placentas were from patients who had PE without severe features and 5 from patients who had PE with severe features such as persistent hypertension (blood pressure above 160/110) and liver enzymes elevated up 


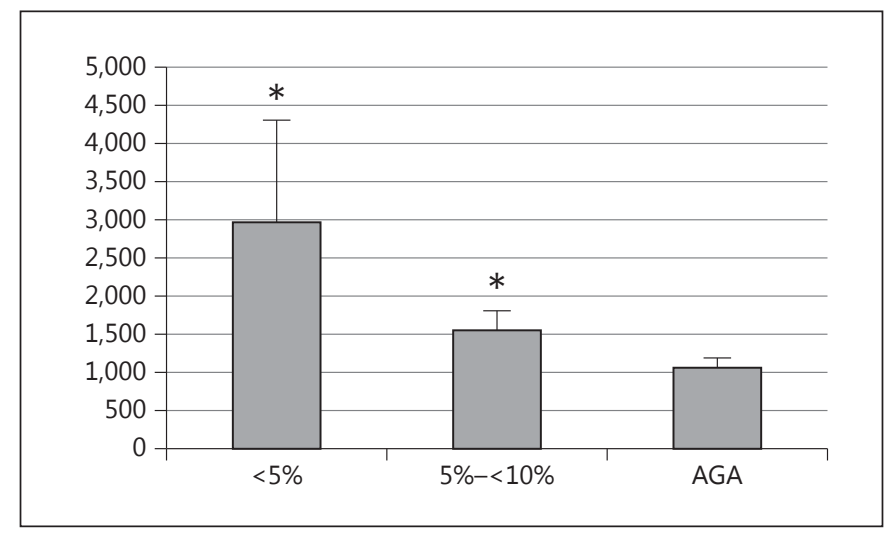

Fig. 1. The CNV burden in FGR. The average amount of bases (kb) with CNVs in the placentas from FGR pregnancies compared to healthy controls. AGA $=$ Appropriate gestational age. ${ }^{*} \mathrm{p}<0.005$.

to twice the upper limits of normal. In 2 samples, the mothers had elevated creatinine (above $1.5 \mathrm{mg} / \mathrm{dl}$ ), and in another, the mother had thrombocytopenia $\left(60,000 / \mathrm{mm}^{3}\right)$. The fetuses of the mothers with PE were all of normal growth (table 1). The placentas from patients with PE were compared to 5 placentas from healthy controls (different samples than those used for the FGR controls).

Placental biopsies were collected within 20 min of delivery under sterile conditions. The biopsies were taken from the intermediate trophoblast midway between the cord insertion and the edge of the placenta and were immediately frozen at $-80^{\circ} \mathrm{C}$.

Genetic Chip Analysis

Thawed placental biopsies were homogenized and genomic DNA was extracted using the Magna Pure Compact Nucleic Acid Isolation kit/Large volume (Roche Diagnostics, Indianapolis, Ind., USA).

Using the Affymetrix GeneChip (Affymetrix, Santa Clara, Calif., USA) for chromosomal microarray (CMA), placental extracts were analyzed for CNVs. The results were analyzed using the Signature Genomics database. We searched for previously described gene functions using the Genome Browser and OMIM database for the genomic coordination of the gene gains and losses detected in the placental analyses. The CNV burden was analyzed and compared among the study groups.

\section{Statistical Analysis}

Nonparametric Kruskal-Wallis and Mann-Whitney U tests were used to analyze the differences between the study groups, with $\mathrm{p}<0.05$ considered statistically significant. SPSS software (SPSS Inc., Chicago, Ill., USA) was used for statistical analysis.

\section{Results}

The relevant clinical characteristics of analyzed patients are listed in table 1. Maternal ages as well as gestational ages at delivery were similar among the groups and in agreement with the accepted treatment protocols for

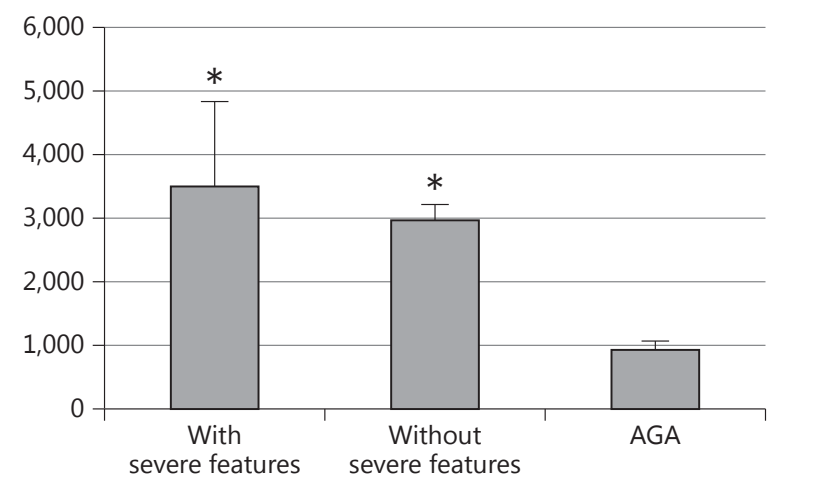

Fig. 2. The CNV burden in PE. The average amount of bases (kb) with CNVs in the placentas from pregnancies with preeclampsia compared to healthy controls. AGA = Appropriate gestational age. $* \mathrm{p}<0.005$.

patients who have PE or pregnancies with FGR. The clinical data and birth weights were consistent with the inclusion criteria for each study group.

CMA analyses identified significantly more genomic alterations among FGR and PE placentas than among healthy controls. Most of the alterations were gene gains (80\%); some had gene deletions (20\%). The analyses included a total of 30 placentas with different clinical pathologies. The genomic alterations were below the resolution of normal karyotyping, and the high variability of the elevated and deleted genes prevented the detection of specific alterations that characterize certain placental pathologies. The altered genes were related to adult height, stress reactions, and cellular migration, differentiation and adhesion.

The CNVs were found in different regions, and the variability between the patients within the study groups was too high to enable analysis based on specific variations. Given the small sample sizes and the variations among the altered genes, we analyzed the CNV burden among the biopsies, i.e. the amount of variations $(\mathrm{kb})$ that were detected by the CMA analysis in each group of samples. This method enabled us to overcome the variability of regions and CNVs among the patients. We found a significant correlation between the CNV burden and the severity of FGR and PE (figs. 1,2).

\section{Discussion}

In the current study, we found increased CNVs in placentas from pregnancies complicated by FGR and PE, both related to abnormal placentation. 
The severity of the diseases and the CNV burden of altered genes were correlated, indicating a genetic basis for both placenta-related FGR and PE.

The role of the placenta in the pathogenesis of FGR as well as in PE is well established [Palloto and Kilbride, 2006; Adams et al., 2014; Friedman and Cleary, 2014]. Therefore, placenta-based investigation of CNVs is crucial for understanding FGR, PE and their related genes and pathways.

Observation of placentas from genetically normal pregnancies reveals a different aspect of the genetic evaluation. CMA analyses enable focusing on gene alterations that are below the detectable levels of known genetic abnormalities [Kearney et al., 2011; Ganesamoorthy et al., 2013]. CMA analysis in the current study revealed heterogenic genetic changes. The small number of samples and the variability of the CNVs among the participants did not allow us to detect specific genes whose enhancement or deletion could potentially be related to placental pathology. Consequently, we looked at the CNV burden and found that it is increased in both FGR and PE pregnancies compared to healthy controls. Further investigation into the specific gene alterations is warranted and requires a larger sample size, which will potentially overcome the variability among the samples.

CMA analysis allows the examination of chromosomes to a resolution of $1 \mathrm{~kb}$, smaller than the average gene. This is an advantage compared to standard karyotyping, when it comes to detecting smaller pathogenic chromosomal variations.

A challenge when employing CMA is revealing chromosomal variants of unknown clinical significance [Kearney et al., 2011]. This technique is now common in prenatal diagnosis, and based on our results, it is pivotal for research regarding placental abnormalities.

In agreement with the results found here, other studies on genetic signatures in FGR and PE revealed typical changes. A case-control comparison between patients with and without PE revealed specific CNVs among the patients with PE. The findings were consistent among 3 different ethnic groups. These data support a genetic predisposition to PE [Zhao et al., 2013]. That study focused on maternal blood. Looking at placental biopsies may reveal new information. Further studies should compare maternal blood to fetal blood and placental samples. CNVs in maternal blood may show a predisposition of PE and even FGR, while placental CNVs may imply on intrauterine programming and later onset diseases.

Placental CNVs are probably a normal feature of trophoblasts. Specific changes occur allowing placental inva- sion and function during pregnancy [Hannibal et al., 2014]. In mice, specific CNVs were shown to be essential for trophoblast invasion and uterine vascular remodeling as a part of maternal adaptation to the pregnancy [Hu and Cross, 2010]. The invasion of trophoblasts into the uterine wall is essential for fetal development and shares common features with tumor progression. However, placental growth regulation, unlike tumor invasion, is precise. Some of the well-known oncogenes and tumor suppressors are involved in abnormal placentation. Despite the heterogeneity, a number of differences in gene expression related to PE were found. These profiles include genes coding for multicellular structure development and differentiation, activity of immune response, inflammation, metabolism-associated proteins, cell proliferation, and apoptosis [Louwen et al., 2012]. Microarray bioinformatic analysis of vascular tissue from 3 placentas associated with FGR identified alterations in genes involved in mitochondrial function, oxidative phosphorylation energy production, and metabolism [Madeleneau et al., 2015].

The importance of the current study is based on having samples from patients with FGR and PE related to placental insufficiency, which were based on accurate clinical data. Furthermore, we show a novel approach to middle cerebral artery evaluation of the placenta.

This study is limited by the small number of placental samples from each group which did not allow interpretation of gene variability. Gaps in information regarding the newborn and the parents prevented complete genetic evaluations. Moreover, the data should be validated by other techniques. Therefore, the findings presented here should be accepted with caution and lead to further research.

To conclude, though very preliminary, the data presented here support placental evaluation using CMA for pregnancies complicated by FGR or PE. Larger data sets are needed for further evaluation of the findings and their clinical implications. Understanding gene alterations related to placental insufficiency may lead to increased understanding of FGR and PE.

\section{Statement of Ethics}

The study was approved by the Institutional Ethics Review Board, and all patients provided signed informed consent.

\section{Disclosure Statement}

The authors have no conflict of interest to declare. 


\section{References}

Adams T, Yeh C, Bennett-Kunzier N, Kinzler WL: Long-term maternal morbidity and mortality associated with ischemic placental disease. Semin Perinatol 38:146-150 (2014).

Barker DJ: Adult consequences of fetal growth restriction. Clin Obstet Gynecol 49:270-283 (2006).

-Dollberg S, Haklai Z, Mimouni FB, Gorfein I, Gordon ES: Birth weight standards in the liveborn population in Israel. Isr Med Assoc J 7: 311-314 (2005).

Durnwald C, Landon M: Fetal links to chronic disease: the role of gestational diabetes mellitus. Am J Perinatol 30:343-346 (2013).

-Founds SA, Conley YP, Lyons-Weiler JF, Jeyabalan A, Hogge WA, Conrad KP: Altered global gene expression in first trimester placentas of women destined to develop preeclampsia. Placenta 30:15-24 (2009).

-Friedman AM, Cleary KL: Prediction and prevention of ischemic placental disease. Semin Perinatol 38:177-182 (2014).

Ganesamoorthy D, Bruno DL, McGillivray G, Norris F, White SM, et al: Meeting the challenge of interpreting high-resolution single nucleotide polymorphism array data in prenatal diagnosis: does increased diagnostic power outweigh the dilemma of rare variants? BJOG 120:594-606 (2013).
Hannibal RL, Chuong EB, Rivera-Mulia JC, Gilbert DM, Valouev A, Baker JC: Copy number variation is a fundamental aspect of the placental genome. PLoS Genet 10:e1004290 (2014).

Hu D, Cross JC: Development and function of trophoblast giant cells in the rodent placenta. Int J Dev Biol 54:341-354 (2010).

Kearney HM, Thorland EC, Brown KK, Quintero-Rivera F, South ST; Working group of the American College of Medical Genetics Laboratory Quality Assurance Committee: American College of Medical Genetics standards and guidelines for interpretation and reporting of postnatal constitutional copy number variation. Genet Med 13:680-685 (2011).

Louwen F, Muschol-Steinmetz C, Reinhard J, Reitter A, Yuan J: A lesson for cancer research: placental microarray gene analysis in preeclampsia. Oncotarget 3:759-773 (2012).

Lupski JR: Genetics. Genome mosaicism-one human, multiple genomes. Science 341:358-359 (2013).

Madeleneau D, Buffat C, Mondon F, Grimault H, Rigourd V, et al: Transcriptomic analysis of human placenta in intrauterine growth restriction. Pediatr Res 77:799-807 (2015).
Pallotto EK, Kilbride HW: Perinatal outcome and later implications of intrauterine growth restriction. Clin Obstet Gynecol 49:257-269 (2006).

Patti ME: Intergenerational programming of metabolic disease: evidence from human populations and experimental animal models. Cell Mol Life Sci 70:1597-1608 (2013).

Rabaglino MB, Post Uiterweer ED, Jeyabalan A, Hogge WA, Conrad KP: Bioinformatics approach reveals evidence for impaired endometrial maturation before and during early pregnancy in women who developed preeclampsia. Hypertension 65:421-429 (2015).

Ross MG, Desai M: Developmental programming of offspring obesity, adipogenesis, and appetite. Clin Obstet Gynecol 56:529-536 (2013).

Sibai BM: Diagnosis, prevention, and management of eclampsia. Obstet Gynecol 105:402410 (2005).

Sibai B, Dekker G, Kupferminc M: Pre-eclampsia. Lancet 365:785-799 (2005).

Zhao L, Bracken MB, DeWan AT: Genome-wide association study of pre-eclampsia detects novel maternal single nucleotide polymorphisms and copy-number variants in subsets of the Hyperglycemia and Adverse Pregnancy Outcome (HAPO) Study Cohort. Ann Hum Genet 77:277-287 (2013). 